\title{
ATTACHMENT FACTOR AND RECEPTOR ENGAGEMENT OF SARS CORONAVIRUS AND HUMAN CORONAVIRUS NL63
}

\author{
Heike Hofmann, Andrea Marzi, Thomas Gramberg, Martina Geier, \\ Krzysztof Pyrc, Lia van der Hoek, Ben Berkhout, and Stefan Pöhlmann*
}

\section{INTRODUCTION}

The cellular membrane constitutes a physical barrier against viral infection. Enveloped viruses developed specialized proteins to overcome this barrier. These proteins, which are often extensively glycosylated, are inserted into the viral membrane and mediate both recognition of target cells and fusion of the viral membrane with a host cell membrane. The latter process allows introduction of the viral genome and associated viral proteins into the host cell lumen and is therefore critical for establishment of productive infection. ${ }^{1,2}$ Because of their important function, the viral membrane glycoproteins, which in the case of coronaviruses $(\mathrm{CoV})$ are termed spike (S) proteins, are attractive targets for inhibitors and vaccines.

Enveloped viruses evolved two prototypes of glycoproteins to enter target cells, termed class I and class II fusion proteins. ${ }^{3,}$ Class I fusion proteins are found in, e.g., retroviruses and paramyxoviruses, while, e.g., flaviviruses and alphaviruses encode class II fusion proteins. Both types of fusion proteins exhibit a distinct functional organization, which is reflected by their different spatial orientations. Thus, class I fusion proteins are oriented perpendicular to the cellular membrane and are visible as spikes in electron micrographs, while class II proteins are oriented horizontally relative to the cellular membrane and are well ordered on the virion surface. Viral class I fusion proteins are organized into a globular surface unit (SU), which interacts with cellular receptors, and a transmembrane unit (TM), which harbors highly conserved sequence elements required for membrane fusion. ${ }^{3}$ Membrane fusion is initiated by binding of SU to cellular receptor(s) or by exposure of the glycoprotein to low $\mathrm{pH}$, which triggers conformational changes in the glycoprotein that activate TM. TM-driven membrane fusion is initiated by insertion of a N-terminal fusion peptide into the target cell membrane, followed by

\footnotetext{
*Heike Hofmann, Andrea Marzi, Thomas Gramberg, Martina Geier, Stefan Pöhlmann, University of ErlangenNürnberg, Germany. Krzysztof Pyrc, Lia van der Hoek, Ben Berkhout, University of Amsterdam, The Netherlands.
} 
conformational changes in TM during which two heptad repeats in the extracellular part of TM fold back onto each other and pull the viral and target cell membranes into close contact, which ultimately promotes membrane fusion. Despite the different functional organization of class II fusion proteins, which, e.g., harbor the fusion peptide in SU, membrane fusion driven by these proteins follows similar principles. ${ }^{5}$

The S-proteins of CoVs, which protrude from the viral membrane and provide virus particles with the typical corona like shape, exhibit the characteristics of class I fusion proteins. ${ }^{1}$ Some S-proteins are cleaved between S1 and S2, ${ }^{1}$ as is the case with human coronavirus OC43 (HCoV-OC43), and cleavage is possibly important for function. In contrast, cleavage of the spike proteins of HCoV-229 and other group I CoVs has not been observed and cleavage of mouse hepatitis virus (MHV) S appears to be cell-type dependent and not essential for function. ${ }^{1}$ The $\mathrm{S} 1$ domains are adapted to bind to specific cellular receptors, and the spike-receptor interaction is the major determinant of viral cell tropism. ${ }^{1}$ Because the S-proteins of CoVs from different groups are, in most cases, adapted to interact with different receptors, they show little sequence conservation. In contrast, the S2 domains share the same task, fusion of viral and cellular membranes, and therefore exhibit considerable sequence homology. ${ }^{1}$

SARS-CoV infects the lower respiratory tract with fatal outcome in about $10 \%$ of infected individuals. ${ }^{6}$ In contrast, infection with HCoV-NL63 does not cause severe disease, but is often associated with bronchiolitis and cold like symptoms. ${ }^{7,8}$ Although SARS-CoV was, mainly due to air travel, spread into 29 different countries in 2003, the majority of cases were observed in Asia. HCoV-NL63 in turn seems to be a globally distributed pathogen, with HCoV-NL63 infections being reported in Europe, Japan, Canada, and Australia. Sequence analysis revealed that also the S-proteins of SARS-CoV and HCoV-NL63 exhibit features of class I fusion proteins. Thus, the S2 subunits contain heptad repeats, a transmembrane domain and a short intracellular domain, ${ }^{1}$ elements found in the S2 subunits of all CoV S-proteins. The S1 subunit of SARS-CoV, which engages angiotensin converting enzyme 2 (ACE2) as a receptor for cellular entry, ${ }^{9}$ shares little sequence homology with other CoV S-proteins. In contrast, the sequence of the S1 subunit of HCoV-NL63 is $56 \%$ identical to $\mathrm{S} 1$ of hCoV-229E, ${ }^{8}$ which employs aminopeptidase N/CD13 for entry into target cells.

We thought to investigate the range of target cells susceptible to SARS-CoV-S and HCoV-NL63-S dependent infection as well as the interaction of the respective S-proteins with cellular membrane proteins and their recognition by sera from infected patients. For these analyses, we employed retroviral reporter viruses pseudotyped with the CoV-Sproteins. These viruses, so called pseudotypes, were generated by cotransfection of 293T cells with a plasmid encoding a retroviral genome, in which the env open reading frame was inactivated and in which a reporter gene was inserted, together with an expression plasmid for the CoV-S-protein to be studied. Such particles harbor the CoV S-protein in their membrane and enter target cells in a S-protein dependent manner. However, once membrane fusion is completed, all processes leading to viral gene expression are dependent on retroviral proteins. Efficiency of infection with pseudotyped viruses can be conveniently quantified because of expression of the reporter gene encoded by the proviral genome. 


\section{IDENTIFICATION OF TARGET CELLS SUSCEPTIBLE TO SARS-CoV-S DRIVEN INFECTION}

We employed HIV-1 derived reporter viruses harboring the S-protein of the SARS$\mathrm{CoV}$ Frankfurt strain to investigate the range of cells permissive to SARS-CoV-S dependent infection. ${ }^{10}$ Pseudotypes bearing the G-protein of the amphotropic vesicular stomatitis virus (VSV) were used as positive control, while viruses without an envelope protein were employed as negative control. Infection of a panel of cell lines revealed that SARS-CoV-S mediates efficient entry into liver (Huh-7, Hep-G2) and kidney (293T) derived cell lines, and the hepatoma cell line Huh-7 was found to be permissive for SARS-CoV replication. ${ }^{10}$ Entry into liver and kidney derived cell lines is in agreement with subsequent studies demonstrating infection of these organs in SARS patients. ${ }^{11}$ In contrast, lymphoid cell lines were refractory to SARS-CoV-S driven infection, and similar observations were made by an independent study examining replication competent SARS-CoV, ${ }^{12}$ suggesting that lymphoid cells might not support SARS-CoV spread in vivo. We next determined if SARS-CoV-S driven cellular entry depends on an acidic environment. To address this question, Huh-7 and 293T cells were preincubated with the lysosomotropic agents ammonium chloride and bafilomycin A1 and infected with SARS-CoV-S, VSV-G or murine leukemia virus (MLV) bearing pseudotypes. In agreement with published data, entry mediated by the MLV glycoprotein was not blocked by lysosomotropic agents, while entry driven by VSV-G was efficiently inhibited in a dose dependent manner. ${ }^{10}$ Infectious entry of SARS-CoV-S bearing pseudotypes was also blocked, indicating that the SARS-CoV-S protein requires a low $\mathrm{pH}$ environment to unfold its fusogenic activity, ${ }^{10}$ an observation confirmed by several independent studies. ${ }^{13,14}$ Finally, we investigated if sera from SARS patients recognize the SARS$\mathrm{CoV}-\mathrm{S}$ protein and neutralize infection. Transient expression of SARS-CoV-S on 293T cells followed by staining of cells with sera from healthy patients or SARS patients revealed that SARS patient sera recognize the S-protein. ${ }^{10}$ Sera from SARS patients but not control sera neutralized SARS-CoV-S dependent infection, ${ }^{10}$ indicating that infected individuals mount a S-specific neutralizing antibody response.

\section{ANALYSIS OF THE INTERACTION BETWEEN SARS-COV-S AND ITS RECEPTOR ACE2}

Studies by Li and colleagues revealed that SARS-CoV employs ACE2 for entry into target cells. ${ }^{9}$ Specifically, the S1 subunit was shown to bind to ACE2, and fusion of SARS-CoV-S expressing cells with ACE2 but not control cells was demonstrated. ${ }^{9}$ Inhibition analysis indicated that replication of SARS-CoV depends on ACE2, and amino acids 318-510 in SARS-CoV-S were shown to function as an independent receptor binding domain. ${ }^{9,15}$ While these studies indicated that ACE2 is an important receptor for SARS-CoV, it was unclear if the virus engages receptors besides ACE2 for infection of target cells. Therefore, we investigated if expression of ACE2 correlates with permissiveness to SARS-CoV-S dependent infection. Analysis of mRNA expression in a panel of cell lines of known susceptibility to SARS-CoV-S mediated entry revealed that SARS-CoV-S bearing pseudotypes infected exclusively cell lines that express ACE2. ${ }^{16}$ 
These findings suggest that ACE2 is of paramount importance for SARS-CoV entry and most likely constitutes the only receptor for SARS-CoV - a finding substantiated by several subsequent studies. ${ }^{12,17}$ The interaction between SARS-CoV-S and ACE2 is an attractive target for therapeutic intervention. Indeed, several studies described peptide inhibitors that mimic the second heptad repeat in the S2 subunit of SARS-CoV-S and block SARS-CoV-S infection in the low micromolar range. ${ }^{1}$ We investigated if inhibitors based on the ACE2 ectodomain might also be effective. To this end, we incubated SARSCoV-S and VSV-G bearing pseudotypes with concentrated soluble ACE2 ectodomain and analysed infection of target cells. The ACE2 ectodomain inhibited SARS-CoV-S in a potent and specific manner, ${ }^{16}$ suggesting that polypeptides based on the ACE2 ectodomain could, at least in theory, be developed as therapeutics. Finally we asked if the cytoplasmic domain of ACE2, which harbors consensus sites for tyrosine kinases and casein kinase II motifs, is required for receptor function. However, ACE2 variants in which the cytoplasmic tail was stepwise deleted were fully capable of promoting SARSCoV-S dependent entry into transiently transfected $293 \mathrm{~T}$ cells, ${ }^{16}$ indicating that the cytoplasmic domain of ACE2 might be dispensable for receptor function - at least under conditions of overexpression in already permissive cells.

\section{ROLE OF CELLULAR LECTINS IN SARS-CoV INFECTION}

Engagement of cellular receptors by viral glycoproteins is essential for virus entry into target cells. However, engagement of cellular factors other than the viral receptor can affect infection efficiency. Thus, binding to so called attachment factors can concentrate viruses on the surface of target cells, thereby increasing the chance of receptor engagement and subsequent infectious entry. ${ }^{18}$ The lectin DC-SIGN is a universal pathogen attachment factor and promotes infection by a variety of viral and non viral pathogens. ${ }^{1,19}$ Maybe most strikingly, DC-SIGN is expressed on dendritic cells (DCs) and facilitates the HIV interaction with these cells, which is believed to be important for viral dissemination. ${ }^{19}$ DC-SIGN binds to high-mannose carbohydrates in the HIV-Env protein and facilitates both infection of the DC-SIGN expressing cells (in case they also express the HIV receptors CD4 and CCR5/CXCR4) and of adjacent susceptible cells. ${ }^{19}$ The former process is termed infection in cis, while the latter process is termed infection in trans or transmission. Apart from DC-SIGN, the related protein DC-SIGNR, for DCSIGNrelated (also termed L-SIGN, for Liver-SIGN), was also shown to function as a pathogen attachment factor. ${ }^{20,21}$ In contrast to DC-SIGN, DC-SIGNR is expressed on sinusoidal endothelial cells in lymph node and liver ${ }^{20,21}$ and might promote spread of HIV-1 and hepatotropic viruses.

Analysis of ACE2 positive cells expressing DC-SIGN, DC-SIGNR, or a control plasmid revealed that both lectins bind to the S1 unit of SARS-CoV-S and augment SARS-CoV-S dependent infection. ${ }^{14,22}$ Importantly, however, expression of DC-SIGN and DC-SIGNR on nonpermissive cells did not allow for readily detectable SARS-CoV-S mediated infectious entry, and DC-SIGN positive immature DCs were refractory to infection, ${ }^{14,22}$ indicating that DC-SIGN and DC-SIGNR function as SARS-CoV attachment factors and not as viral receptors. DC-SIGN expressing, nonpermissive cells 
and DCs transmitted SARS-CoV-S bearing pseudotypes and replication competent SARS-CoV to adjacent permissive cells, ${ }^{14,22}$ indicating that DCs might promote SARS$\mathrm{CoV}$ dissemination in infected individuals. Similarly, DC-SIGNR expression in the lung might promote SARS-CoV spread in this major target organ. ${ }^{23}$

\section{IDENTIFICATION OF ACE2 AS A RECEPTOR FOR HCoV-NL63}

\subsection{Pseudotypes Bearing the S-Proteins of HCoV-NL63-S and SARS-CoV-S Exhibit a Comparable Cell Tropism}

Monkey kidney cells were shown to be permissive for HCoV-NL63 infection, ${ }^{7,8}$ however, the range of susceptible target cells had not been identified. In order to analyze the cellular tropism of HCoV-NL63, we employed lentiviral pseudotypes carrying the Sprotein of HCoV-NL63 and included virions pseudotyped with SARS-CoV-S and HCoV$229 \mathrm{E}-\mathrm{S}$ as controls. The latter S-protein is highly homologous to the NL63-S-protein and it has been suggested that both might employ the same cellular receptor for entry and might thus enter the same target cells. ${ }^{8}$ However, we observed a striking difference in the cell tropism of 229E-S and NL63-S bearing pseudotypes (Table 1), as HOS, MRC-5 and feline FCWF cells were susceptible to 229E-S but not NL63-S-driven infection, whereas only NL63-S mediated entry into 293 T kidney cells. ${ }^{24}$ Interestingly, the cell tropism of NL63-S harboring pseudotypes matched that previously described for SARS-CoV-S bearing pseudovirions, ${ }^{10,13,14}$ suggesting that both S-proteins might engage the same cellular factors for entry. ${ }^{24}$ We next addressed if NL63-S bearing pseudotypes reflect the cell tropism of replication competent HCoV-NL63. Huh-7 cells were highly permissive to NL63-S and SARS-CoV-S driven infection ${ }^{10,24}$ and we therefore expected HCoVNL63 to replicate in these cells. Indeed, four to five days after inoculation with HCoVNL63, a cytopathic effect was readily visible in the infected culture in comparison with mock-infected cells, ${ }^{24}$ indicating that the hepatic cell tropism of NL63-S bearing pseudoparticles is reflected by replication-competent HCoV-NL63.

Table 1. Analysis of the cell tropism of CoV-S-pseudotypes. The indicated cell lines were infected with lentiviral pseudotypes carrying the S-proteins of hCoV-229E, -NL63, and SARS-CoV and reporter gene activities in cellular lysates quantified. Infection efficiency is shown as: (-), no infection, $(+)$, low, $(+++)$ high.

\begin{tabular}{|l|l|l|l|l|}
\hline Cell type & Cell line & 229E-S & NL63-S & SARS-S \\
\hline T-lymphocyte & C81-66 & - & - & - \\
\hline & CEMx174 & - & - & - \\
\hline B-lymphocyte & BL41 & - & - & - \\
\hline Kidney & $293 \mathrm{~T}$ & - & + & + \\
\hline Fibroblast & HFF & + & - & n.d. \\
\hline & MRC-5 & +++ & - & - \\
\hline Fibroblast (feline) & FCWF & +++ & - & - \\
\hline Glioblastoma & U373 & - & - & - \\
\hline Epithelial & Hela S3 & - & - & - \\
\hline & Hep-2 & - & + & + \\
\hline Osteosarcoma & HOS & +++ & - & - \\
\hline Liver & HepG2 & + & + & + \\
\hline & Huh7 & +++ & +++ & +++ \\
\hline
\end{tabular}




\subsection{HCoV-NL63-S Dependent Entry Requires Low pH}

Because NL63-S and SARS-CoV-S bearing pseudotypes exhibited a comparable cell tropism, we next asked if entry mediated by both S-proteins depends on low $\mathrm{pH}$. To address this question, Huh-7 target cells were incubated with the lysosomotropic agents bafilomycin A1 or ammonium chloride and infected with pseudovirions bearing VSV-G, MLV, and the spike proteins of HCoV-NL63, HCoV-229E, and SARS-CoV (data not shown). As expected, VSV-G driven entry was inhibited by the lysosomotropic agents, while MLV glycoprotein dependent entry was not affected. Entry mediated by all CoV Sproteins examined was dependent on low $\mathrm{pH}$, suggesting that SARS-CoV, HCoV-NL63 and $\mathrm{HCoV}-229 \mathrm{E}$ employ the same route of entry.

\subsection{The S-Protein of HCoV-NL63 Engages ACE2 but Not CD13 for Cellular Entry}

Feline CD13 (fCD13) serves as a receptor for all CoVs of the phylogenetic group I. Because HCoV-NL63-S is a group I virus, it was expected that NL63-S might also engage fCD13 for entry. In the HCoV-NL63 cell tropism experiments described above, however, we observed that the feline FCWF cells, which express fCD13, were refractory to NL63-S mediated infection. Similarly, 293T cells overexpressing fCD13 or human CD13 were permissive to 229E-S mediated infection, while expression of both feline or human CD13 did not augment infection driven by NL63-S, ${ }^{24}$ indicating that CD13 is not involved in HCoV-NL63 entry. Because the cellular tropism of NL63-S and SARS-CoV$\mathrm{S}$ bearing pseudotypes was identical, we next investigated if the SARS-CoV receptor ACE2 plays a role in NL63-S dependent entry. To address this question, we transiently expressed ACE2 on 293 T cells and over-infected the cells with 229E-, NL63- or SARS-CoV$\mathrm{S}$ bearing pseudotypes. Hereby, a significant enhancement of infection could be documented for NL63-S- and SARS-CoV-S-, but not for 229E-S-bearing pseudotypes. ${ }^{24}$ The interaction of NL63-S and ACE2 was specific, as expression of the closely related ACE1 protein did allow for augmentation of infection. ${ }^{24}$ Additionally, we were able to inhibit both NL63-S mediated entry and replication of HCoV-NL63 by an ACE2-specific antiserum, but not by antibodies directed against $\mathrm{ACE} 1,{ }^{24}$ confirming the specificity of the interaction. Finally, we employed soluble NL63-S1 and SARS-CoV-S1 proteins to investigate if NL63-S directly interacts with ACE2. Both NL63-S and SARS-CoV-S bound efficiently to 293T cells expressing ACE2 but not control cells, ${ }^{24}$ indicating that NL63-S directly contacts ACE2.

We next addressed the question whether ACE2 alone is sufficient to mediate $\mathrm{HCoV}$ NL63 entry. For this, we transiently expressed ACE2 on nonpermissive Hela cells followed by infection with NL63- or SARS-S bearing pseudotypes. Whereas the presence of ACE2 allowed for efficient SARS-S-mediated entry, we observed only a slight enhancement of NL63-S dependent entry (data not shown). This finding can be explained in three ways: (i) SARS-S exhibits a higher affinity for ACE2 than NL63-S, (ii) a much higher amount of ACE2 has to be present on a target cell for efficient NL63-S mediated entry compared to SARS-CoV-S driven infection, or (iii) HCoV-NL63 requires a so far unidentified co-receptor which is not or only insufficiently present on Hela cells. Additionally, it is possible that a cellular factor involved in steps after receptor engagement might be critical for NL63-S mediated infection, but could be dispensable for SARS$\mathrm{S}$ dependent cellular entry. Further experiments are required to decipher these differences between HCoV-NL63 and SARS-CoV entry into target cells. 


\subsection{Evidence That HCoV-NL63 Infection Is Common and Usually Acquired During Childhood}

Initial PCR-based screening experiments suggested that HCoV-NL63 infection is relatively frequent, ${ }^{7,8}$ but serological data were not included in these studies. Employing NL63-S bearing pseudoparticles, we investigated whether adults with or without respiratory tract illness exhibit a neutralizing antibody response against NL63-S. Interestingly, we found strong neutralizing activities against NL63-S, but not 229E-S in the sera of all adults tested. ${ }^{24}$ Therefore, HCoV-NL63 infection seems to be frequent and more prevalent than infection with hCoV-229E. Furthermore, sera that neutralized NL63$\mathrm{S}$ dependent infection did not necessarily block $229 \mathrm{E}-\mathrm{S}$ mediated infectious entry, ${ }^{24}$ suggesting that no cross-reactive antibodies are induced in infected individuals, despite the high amino acid similarity between both S-proteins. When investigating sera from children of different age groups, we observed that a neutralizing antibody response against NL63-S can first be detected at the age of 1.5 years and is found in all samples from donors aged at least 8 years. ${ }^{24}$ In contrast, none of the sera investigated showed reactivity against SARS-CoV, indicating that NL63-S and SARS-CoV-S, despite using the same receptor for cellular entry, do not share determinants recognized by neutralizing antibodies. $^{24}$

\section{CONCLUDING REMARKS}

We employed retroviral pseudotypes to analyze the S-proteins of SARS-CoV and HCoV-NL63. Our analysis, and its comparison with independent studies using replication competent CoVs, show that S-protein bearing pseudotypes adequately reflect cell tropism, receptor and attachment factor usage and route of entry of the CoVs from which the S-proteins were derived. We found that SARS-CoV-S bearing pseudotypes infect a relatively broad range of cells, ${ }^{10}$ leading us to the conclusion that SARS-CoV might target organs other than the lung in infected patients. Indeed, subsequent studies examining tissues from SARS patients confirmed that SARS-CoV targets a variety of organs. ${ }^{11}$ Entry of SARS-CoV-S pseudotypes ${ }^{10,13,14}$ and replication competent virus ${ }^{25}$ can be inhibited by compounds that impede acidification of the endosomal compartment, suggesting that low $\mathrm{pH}$ might trigger structural rearrangements in SARS-CoV-S pivotal to membrane fusion. However, a subsequent study revealed that an acidic environment is required for the activity of cellular cathepsin proteases, which cleave the SARS-CoV-S, and possibly the NL63-S-protein, and cleavage was found to be required for infectious cellular entry. ${ }^{25}$ Thus, SARS-CoV-S dependent entry follows a novel principle, and offers new targets for therapeutic intervention. ${ }^{25}$ Entry of SARS-CoV was strictly dependent on expression of ACE2, ${ }^{16}$ indicating that ACE2 is the only cellular receptor for SARS-CoV. The ACE2 ectodomain was found to inhibit SARS-CoV-S dependent entry ${ }^{16}$ and it might be possible to generate ACE2 derived inhibitors. Compounds based on the ACE2 ectodomain might be particularly promising, as it has been proposed that downmodulation of ACE2 during SARS-CoV infection is responsible for much of the SARS pathology, which can be prevented by application of the soluble ACE2 ectodomain. ${ }^{26}$ While ACE2 promotes entry of SARS-CoV, viral entry is enhanced by S-protein binding to the lectins DC-SIGN and DC-SIGNR. ${ }^{14,22}$ Our studies indicate that DC-SIGN and DC- 
SIGNR augment infection but do not allow for infectious entry in the absence of ACE2. ${ }^{10}$ However, another study employing replication competent SARS-CoV reported that DCSIGNR functions as a viral receptor. ${ }^{23}$ In any case, it will be interesting to analyze if polymorphisms in the DC-SIGNR neck region, which are frequently found, modulate the risk or outcome of SARS-CoV infection. SARS-CoV shares ACE2 as a receptor and DCSIGN/DC-SIGNR as attachment factors with the group I HCoV-NL63. ${ }^{24}$ The observation that HCoV-NL63 employs ACE2 for infection and consequently enters the same target cells as SARS-CoV, but does not induce severe disease, poses a variety of interesting questions. Are the accessory genes responsible of these differences in pathogenicity, nine of which are present in SARS-CoV compared with only one in NL63? Do SARS-CoV-S and NL63-S engage ACE2 differentially, and are potential differences associated with differences in pathogenicity? Is HCoV-NL63-S sensitive to ACE2 inhibitors? Can a chimeric NL63-S/229E-S protein be generated that induces neutralizing antibodies against both viruses and can be developed as a vaccine? Is ACE2 the only receptor for HCoV-NL63? While experiments with transiently transfected non permissive HeLa cells indeed suggest that HCoV-NL63 could use a coreceptor for entry (data not shown), a thorough comparative analysis of SARS-CoV and HCoV-NL63 infectious entry, including the establishment of animal models for HCoV-NL63 infection, is required to answer these questions.

\section{REFERENCES}

1. H. Hofmann and S. Pöhlmann, Cellular entry of the SARS coronavirus, Trends Microbiol. 12, 466-472 (2004).

2. A. E. Smith and A. Helenius, How viruses enter animal cells, Science 304, 237-242 (2004).

3. D. M. Eckert and P. S. Kim, Mechanisms of viral membrane fusion and its inhibition, Annu. Rev. Biochem. 70, $777-810(2001)$

4. F. X. Heinz, K. Stiasny, and S. L. Allison, The entry machinery of flaviviruses, Arch. Virol. Suppl. (18), 133-137 (2004).

5. D. J. Schibli and W. Weissenhorn, Class I and class II viral fusion protein structures reveal similar principles in membrane fusion, Mol. Membr. Biol. 21, 361-371 (2004).

6. J. S. Peiris, K. Y. Yuen, A. D. Osterhaus, and K. Stohr, The severe acute respiratory syndrome, N. Engl. J. Med. 349, 2431-2441 (2003)

7. R. A. Fouchier, N. G. Hartwig, T. M. Bestebroer, B. Niemeyer, J. C. de Jong, J. H. Simon, and A. D. Osterhaus, A previously undescribed coronavirus associated with respiratory disease in humans, Proc. Natl. Acad. Sci. USA 101, 6212-6216 (2004).

8. L. van der Hoek, K. Pyrc, M. F. Jebbink, W. Vermeulen-Oost, R. J. Berkhout, K. C. Wolthers, P. M. Wertheim-van Dillen, J. Kaandorp, J. Spaargaren, and B. Berkhout, Identification of a new human coronavirus, Nat. Med. 10, 368-373 (2004).

9. W. Li, M. J. Moore, N. Vasilieva, J. Sui, S. K. Wong, M. A. Berne, M. Somasundaran, J. L. Sullivan, K. Luzuriaga, T. C. Greenough, H. Choe, and M. Farzan, Angiotensin-converting enzyme 2 is a functional receptor for the SARS coronavirus, Nature 426, 450-454 (2003).

10. H. Hofmann, K. Hattermann, A. Marzi, T. Gramberg, M. Geier, M. Krumbiegel, S. Kuate, K. Uberla, M Niedrig, and S. Pöhlmann, S protein of severe acute respiratory syndrome-associated coronavirus mediates entry into hepatoma cell lines and is targeted by neutralizing antibodies in infected patients, J. Virol. 78 , 6134-6142 (2004).

11. J. Gu, E. Gong, B. Zhang, J. Zheng, Z. Gao, Y. Zhong, W. Zou, J. Zhan, S. Wang, Z. Xie, H. Zhuang, B. Wu, H. Zhong, H. Shao, W. Fang, D. Gao, F. Pei, X. Li, Z. He, D. Xu, X. Shi, V. M. Anderson, and A. S. Leong, Multiple organ infection and the pathogenesis of SARS, J. Exp. Med. 202, 415-424 (2005).

12. K. Hattermann, M. A. Muller, A. Nitsche, S. Wendt, M. O. Donoso, and M. Niedrig, Susceptibility of different eukaryotic cell lines to SARS-coronavirus, Arch. Virol. 150, 1023-1031 (2005). 
13. G. Simmons, J. D. Reeves, A. J. Rennekamp, S. M. Amberg, A. J. Piefer, and P. Bates, Characterization of severe acute respiratory syndrome-associated coronavirus (SARS-CoV) spike glycoprotein-mediated viral entry, Proc. Natl. Acad. Sci. USA 101, 4240-4245 (2004).

14. Z. Y. Yang, Y. Huang, L. Ganesh, K. Leung, W. P. Kong, O. Schwartz, K. Subbarao, and G. J. Nabel, pHdependent entry of severe acute respiratory syndrome coronavirus is mediated by the spike glycoprotein and enhanced by dendritic cell transfer through DC-SIGN, J. Virol. 78, 5642-5650 (2004).

15. S. K. Wong, W. Li, M. J. Moore, H. Choe, and M. Farzan, A 193-amino acid fragment of the SARS coronavirus S protein efficiently binds angiotensin-converting enzyme 2, J. Biol. Chem. 279, 3197-3201 (2004).

16. H. Hofmann, M. Geier, A. Marzi, M. Krumbiegel, M. Peipp, G. H. Fey, T. Gramberg, and S. Pöhlmann, Susceptibility to SARS coronavirus S protein-driven infection correlates with expression of angiotensin converting enzyme 2 and infection can be blocked by soluble receptor, Biochem. Biophys. Res. Commun. 319, 1216-1221 (2004).

17. E. C. Mossel, C. Huang, K. Narayanan, S. Makino, R. B. Tesh, and C. J. Peters, Exogenous ACE2 expression allows refractory cell lines to support severe acute respiratory syndrome coronavirus replication, J. Virol. 79, 3846-3850 (2005).

18. S. Ugolini, I. Mondor, and Q. J. Sattentau, HIV-1 attachment: another look, Trends Microbiol. 7, 144-149 (1999).

19. Y. van Kooyk and T. B. Geijtenbeek, DC-SIGN: escape mechanism for pathogens, Nat. Rev. Immunol. 3 697-709 (2003).

20. A. A. Bashirova, T. B. Geijtenbeek, G. C. van Duijnhoven, S. J. van Vliet, J. B. Eilering, M. P. Martin, L. Wu, T. D. Martin, N. Viebig, P. A. Knolle, V. N. KewalRamani, Y. van Kooyk, and M. Carrington, A dendritic cell-specific intercellular adhesion molecule 3-grabbing nonintegrin (DC-SIGN)-related protein is highly expressed on human liver sinusoidal endothelial cells and promotes HIV-1 infection, J. Exp. Med. 193, 671-678 (2001).

21. S. Pöhlmann, E. J. Soilleux, F. Baribaud, G. J. Leslie, L. S. Morris, J. Trowsdale, B. Lee, N. Coleman, and R. W. Doms, DC-SIGNR, a DC-SIGN homologue expressed in endothelial cells, binds to human and simian immunodeficiency viruses and activates infection in trans, Proc. Natl. Acad. Sci. USA 98, 2670-2675 (2001).

22. A. Marzi, T. Gramberg, G. Simmons, P. Moller, A. J. Rennekamp, M. Krumbiegel, M. Geier, J. Eisemann, N. Turza, B. Saunier, A. Steinkasserer, S. Becker, P. Bates, H. Hofmann, and S. Pöhlmann, DC-SIGN and DC-SIGNR interact with the glycoprotein of Marburg virus and the S protein of severe acute respiratory syndrome coronavirus, J. Virol. 78, 12090-12095 (2004).

23. S. A. Jeffers, S. M. Tusell, L. Gillim-Ross, E. M. Hemmila, J. E. Achenbach, G. J. Babcock, W. D. Thomas, Jr., L. B. Thackray, M. D. Young, R. J. Mason, D. M. Ambrosino, D. E. Wentworth, J. C. Demartini, and K. V. Holmes, CD209L (L-SIGN) is a receptor for severe acute respiratory syndrome coronavirus, Proc. Natl. Acad. Sci. USA 101, 15748-15753 (2004).

24. H. Hofmann, K. Pyrc, H. L. van der, M. Geier, B. Berkhout, and S. Pöhlmann, Human coronavirus NL63 employs the severe acute respiratory syndrome coronavirus receptor for cellular entry, Proc. Natl. Acad. Sci. USA 102, 7988-7993 (2005).

25. G. Simmons, D. N. Gosalia, A. J. Rennekamp, J. D. Reeves, S. L. Diamond, and P. Bates, Inhibitors of cathepsin L prevent severe acute respiratory syndrome coronavirus entry, Proc. Natl. Acad. Sci. USA 102, 11876-11881 (2005).

26. K. Kuba, Y. Imai, S. Rao, H. Gao, F. Guo, B. Guan, Y. Huan, P. Yang, Y. Zhang, W. Deng, L. Bao, B. Zhang, G. Liu, Z. Wang, M. Chappell, Y. Liu, D. Zheng, A. Leibbrandt, T. Wada, A. S. Slutsky, D. Liu, C. Qin, C. Jiang, and J. M. Penninger, A crucial role of angiotensin converting enzyme 2 (ACE2) in SARS coronavirus-induced lung injury, Nat. Med. 11, 875-879 (2005). 